\title{
The Lagrange variety approach applied to frustrated classical wheels
}

\author{
W. Florek ${ }^{1}$, A. Marlewski ${ }^{2}$, G. Kamieniarz ${ }^{1}$, M. Antkowiak ${ }^{1}$ \\ ${ }^{1}$ Adam Mickiewicz University, Faculty of Physics, \\ ul. Uniwersytetu Poznańskiego 2, 61-614 Poznań, Poland \\ ${ }^{2}$ Poznań University of Technology, Institute of Mathematics, \\ ul. Piotrowo 3A, 60-965 Poznań, Poland \\ wojciech.florek@amu.edu.pl
}

PACS 75.10.Hk, 75.50.Ee, 75.50.Xx, 02.40.Re

DOI 10.17586/2220-8054-2020-11-1-30-35

The Lagrange variety approach introduced by Schmidt and Luban [J. Phys. A: Math. Gen. 36, 6351 (2003)] is applied to geometrically frustrated wheels (centered regular polygons). It is shown that the lowest energy configurations are planar or collinear. The latter one, characteristic for nonfrustrated classical systems, is also observed in the presence of competing interactions in a well-determined range $\left(0, \alpha_{\mathfrak{c}}\right)$ of the energy function parameter $\alpha$. The 'critical' value $\alpha_{\mathrm{c}}=1 / 4$ is universal, i.e., it does not depend on a system size. In this domain, the geometric frustration is present, but there is no non-trivial degeneracy.

Keywords: frustration, classical spin models, magnetic molecules, Lagrange variety.

Received: 29 December 2019

\section{Introduction}

Ring-shaped magnetic molecules play an important role due to possible realization of single molecule magnets or molecular qubits [1-6]. Moreover, these, with an odd number of spins and dominant antiferromagnetic couplings, are interesting subjects in the study of the spin frustration [7-14]. In this work, we focus on the classical counterparts of rings with an extra spin placed at its center (see Fig. 1) [13-18]. In general, heterometallic systems may be considered [19], but this short paper is limited to homogeneous ones. To avoid frustration in a peripheral ring only centered polygons $\mathrm{XY}_{2 q}$ (corresponding to wheel graphs $W_{2 q+1}$ [20]) are investigated. The aim of this paper is to determine the lowest energy configuration (LEC) for a system of classical spin vectors coupled by the isotropic Heisenberg interactions with two exchange integrals $J$ and $J_{0}$ for the nearest-neighbor spins $s_{j}, 1 \leq j \leq N$, in the peripheral ring and for their couplings with the central spin $s_{0}$, respectively; competing interactions are present for antiferromagnetic couplings $J$, despite the value of $J_{0}$.

(a)

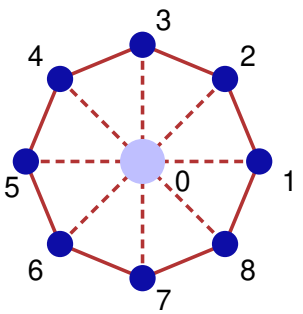

(b)

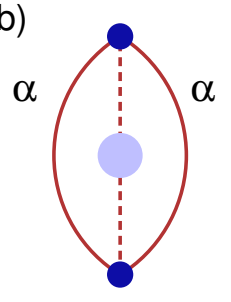

(c)

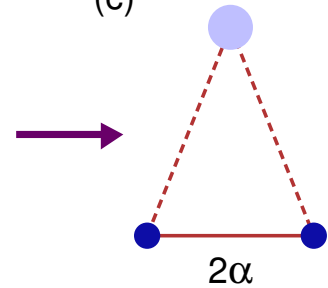

FIG. 1. (a) A centered octagon (the wheel $W_{9}$ ) and (b) a centred regular digon (the 'wheel' $W_{3}$ ) transformed to an isosceles triangle; the weight of the edge $(1,2)$ is doubled to mimic two pairs in the original system. The edges correspond to couplings $J$ and $J_{0}$ (solid and dashed lines, respectively).

Such a system is another example of models in which the collinear LEC is 'retained' when competing interactions are 'turned on' by a relatively weak antiferromagnetic coupling(s) [13,14,21]. This feature can be considered as the classical counterpart of the third type frustration according to the classification scheme recently proposed [8]. To clarify relations among the notions of 'frustration', 'degeneracy', and 'competing interactions', a short resume is given in the next section.

To determine LECs one has to find the global local minimum of energy function, which is a real-valued function of many variables. This is very challenging task and it is difficult to obtain some general results (cf. [22-24]). An efficient method based on the Lagrange variety has been proposed [25] and extensively discussed in a series of papers [26]. This technique enables precise determination of the global minimum and the corresponding LEC considering a general 
form of the energy function for any system size [27]. Such approach has been successfully applied to heterometallic wheels [19] and some of the results obtained are shortly presented below.

This work starts with a short discussion on the frustration and degeneracy. A model considered and solutions obtained are presented in Sec. 3. In the last section the most important conclusions and some challenges are gathered.

\section{Frustration and degeneracy}

The term 'frustration' was introduced by Toulouse [28,29], though spin glasses and other systems with frustration (competing interactions) had been investigated many years earlier [30,31]. Toulouse considered the standard Ising model, i.e., spins $S_{j}= \pm 1$ and exchange integrals $J= \pm 1$. He introduced the frustration function as a product of exchange integrals over a contour $c(J=1$ corresponds to antiferromagnetic couplings)

$$
\Phi(c)=\prod_{\text {contour } c}\left(-J_{j j^{\prime}}\right)
$$

where $j$ and $j^{\prime}$ are labels of neighbouring nodes in the contour considered - the frustration is present if $\Phi(c)=-1$ [28]. Four obvious facts may be established:

- This property characterises a cycle (a system), not an individual spin.

- Antiferromagnetic couplings must be present. To be more precise - the considered cycle has to comprise an odd number of them.

- The ground state (GS) of a system is degenerated.

- Competing interactions are present, i.e., not all bonds (terms in the energy function) are 'satisfied': The GS energy is greater than a sum of the minimum energies of individual bonds (terms).

When more general cases of spin glasses have been considered (e.g., these with random values of $J_{j j^{\prime}} \in \mathbb{R}$ ), another characteristic has been introduced (cf., e.g., [32]):

$$
P(c)=(-1)^{N_{\mathrm{AFM}}},
$$

where $N_{\mathrm{AFM}}$ denotes a number of antiferromagnetic couplings in the contour $c$. Its advantage is to give values restricted to \pm 1 , though, in general, $\left|J_{j j^{\prime}}\right| \neq 1$. However, it 'predicts' frustration $(P(c)=-1)$ in the presence of competing interactions but without the degeneracy (cf. [33]). It can be easily seen in the classical example of an antiferromagnetic triangle $\left(S_{j}= \pm 1\right)$ with the energy function

$$
E=S_{2}\left(S_{1}+S_{3}\right)+\alpha S_{1} S_{3}
$$

According to the rule 'The enemy of my enemy is my ally', in the GS configuration there is $S_{1}=S_{3}$ if $0<\alpha<1$, though these spins are 'enemies' for positive $\alpha$. Note, that this configuration is identical to that of the non-frustrated system with $\alpha<0$ (when $S_{1}$ and $S_{3}$ are in fact 'allies'). The GS degeneracy is present for $\alpha \geq 1$ only. Hence, in the range $0<\alpha<1$ there are merely competing interactions (the term $\alpha S_{1} S_{3}$ is not satisfied), but the degeneracy is absent. This feature can be considered as a counterpart of the third type frustration $[8,13,14]$ for the Ising model.

This phenomenon is absent in some systems. For example, considering the Ising antiferromagnetic pentagon with the energy function (cf. Fig. 2):

$$
E=S_{3}\left(S_{2}+S_{4}\right)+\alpha\left(S_{1} S_{2}+S_{4} S_{5}+S_{1} S_{5}\right)
$$

one obtains that the GS configuration changes at $\alpha=0$ and $\alpha=1$ (cf. Fig. 2) and for all $\alpha>0$ the competing interactions and the degeneracy are present simultaneously.

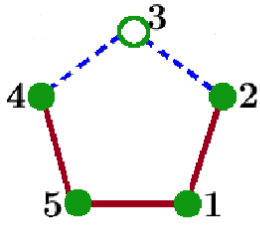

(a)

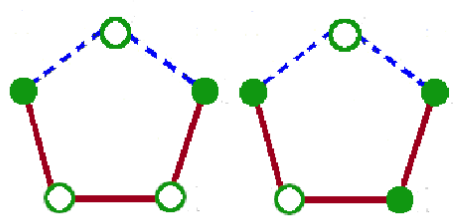

(b)

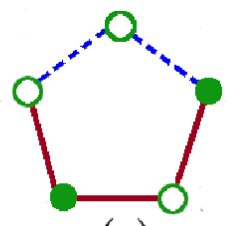

(c)

FIG. 2. The GS configurations for the energy functions given in Eq. (4). The exchange integrals are $J=1$ (dashed lines) or $J=\alpha$ (solid ones); $S= \pm 1$ are denoted by full and empty symbols, respectively. The GS configurations for (a) $\alpha \leq 0$, (b) $0 \leq \alpha \leq 1$, and (c) $\alpha \geq 1$ are presented. Due to lack of the reflection symmetry the two right-most configurations are doubly degenerate. 


\section{Model and its solution}

\subsection{General remarks}

When lengths of spin vectors are fixed (a constraint $\left|s_{j}\right|=1$ for $0 \leq j \leq N$ is assumed in this work), the energy is a real-valued of angles determining positions of vectors $s_{j}$ in $\mathbb{R}^{3}$. The first two vectors $(j=0,1)$ can be fixed to lie in the $x y$ plane, so it is assumed that

$$
\boldsymbol{s}_{0}=[1,0,0], \quad \boldsymbol{s}_{1}=\left[\cos \varphi_{1}, \sin \varphi_{1}, 0\right] .
$$

For the others $(2 \leq j \leq N)$ one may put

$$
\boldsymbol{s}_{j}=\left[\sin \vartheta_{j} \cos \varphi_{j}, \sin \vartheta_{j} \sin \varphi_{j}, \cos \vartheta_{j}\right] .
$$

Hence, in a general case the energy depends on $2 N-1$ variables.

The LEC is collinear for non-frustrated systems [34]. Hence, if the LEC is planar or even spatial then competing interactions are certainly present $[25,34]$. However, these statements do not exclude the special case when competing interactions are present but there is no degeneracy and the LEC is collinear, what can be considered as an analog of the third type frustration in quantum systems.

This possibility is realised in an isosceles antiferromagnetic triangle presented in Fig. 1(b) with the energy function:

$$
E\left(\varphi_{1}, \varphi_{2}\right)=\cos \varphi_{1}+\cos \varphi_{2}+2 \alpha \cos \left(\varphi_{2}-\varphi_{1}\right)
$$

where it is assumed that the LEC is planar (cf. $[13,14,19,34])$. It is easy to show that:

- For $\alpha \leq 1 / 4$ the LEC is collinear with $\varphi_{1}=\varphi_{2}=\pi$ and $E_{1}=2(\alpha-1)$;

- For $\alpha \geq 1 / 4$ the LEC is planar with $\varphi_{2}=-\varphi_{1}, \cos \varphi_{1}=-1 / 4 \alpha$ (so for increasing $\alpha$ the angle $\varphi_{1}$ increases from $\pi$ to $3 \pi / 2$ or decreases to $\pi / 2)$, and $E_{2}=-\left(1+8 \alpha^{2}\right) /(4 \alpha)$.

Therefore, in a range $0<\alpha<1 / 4$ the LEC is collinear in the presence of competing interactions $\left(\boldsymbol{s}_{1}=\boldsymbol{s}_{2}\right.$ despite antiferromagnetic coupling).

\subsection{The Lagrange variety approach}

This approach has been extensively discussed in Schmidt's works [26] and its application to heterogeneous systems have been recently presented [19], so only some important points are mentioned below. In a present version this approach can be applied to isotropic bilinear interactions when a general form of the energy function is given as:

$$
\mathcal{E}=\frac{1}{2} \sum_{(j, k)} J_{j k} \boldsymbol{s}_{j} \cdot \boldsymbol{s}_{k}, \quad J_{j j}=0 .
$$

Exchange integrals $J_{j k}, j \neq k$, are considered as off-diagonal elements of the so-called 'dressed matrix' $\mathbb{J}(\boldsymbol{\lambda})$. Its diagonal elements are determined by a gauge vector:

$$
\boldsymbol{\lambda}=\left[\lambda_{0}, \lambda_{1}, \ldots, \lambda_{N}\right],
$$

with the constraint $\sum_{j} \lambda_{j}=0$. To determine the LEC the least eigenvalue of $\mathbb{J}(\boldsymbol{\lambda})$ has to be found and expressed as a function in $N+1$ variables $\lambda_{j}$. At first glance it seems that this approach is even more difficult than standard analytical methods. However, the gauge vector coefficients have to obey the symmetry of the original problem, so a number of independent parameters is significantly reduced [19,25,26].

The lowest energy and the corresponding LEC (or LECs) of a given system are determined in three steps:

(1) Solve the eigenproblem for the matrix $\mathbb{J}$.

(2) Determine the function $j_{\min }(\boldsymbol{\lambda})$ : a dependence of the minimum eigenvalue with respect to the gauge vector $\boldsymbol{\lambda}$.

(3) The maximum of this function, $\bar{j}=\max _{\boldsymbol{\lambda}} j_{\min }(\boldsymbol{\lambda})$, determines the appropriate gauge vector $\bar{\lambda}$, i.e., $\bar{j}=j_{\min }(\overline{\boldsymbol{\lambda}})$. This specific eigenvalue $\bar{j}$ of $\mathbb{J}(\boldsymbol{\lambda})$ is used to calculate $E_{\text {LEC }}=(N+1) \bar{j} / 2[19,25]$. Some important notes have to be made

- This solution is unique, i.e., there is only one global maximum of $j_{\min }$, but the corresponding eigenvalue may be degenerate.

- This degeneracy $d$ determines a spatial dimension of the LEC: $d=1$ means collinear one, if $d=2$ then the LEC is coplanar etc; with nonphysical solutions for $d>3$ [26].

- In a simplified approach presented here eigenvectors of $\mathbb{J}$ are not necessary and the LECs can be determined solving a system of linear equations [19]. 


\subsection{Classical wheels}

The original energy function (cf. Fig. 1(a)) is given as: $(N+1 \equiv 1)$

$$
\mathcal{E}=J \sum_{j=1}^{N} \boldsymbol{s}_{j} \cdot \boldsymbol{s}_{j+1}+J_{0} \boldsymbol{s}_{0} \cdot \sum_{j=1}^{N} \boldsymbol{s}_{j}
$$

can be rewritten as:

$$
E(\alpha)=\frac{\mathcal{E}}{\left|J_{0}\right|}=\alpha \sum_{j=1}^{N} \boldsymbol{s}_{j} \cdot \boldsymbol{s}_{j+1}+\varepsilon \boldsymbol{s}_{0} \cdot \sum_{j=1}^{N} \boldsymbol{s}_{j}, \quad \alpha=\frac{J}{\left|J_{0}\right|}, \quad \varepsilon=\frac{J_{0}}{\left|J_{0}\right|}= \pm 1 .
$$

The cyclic symmetry demands $\lambda_{j}=\lambda$ for $1 \leq j \leq N$, so a traceless matrix is obtained if $\lambda_{0}=-N \lambda$. Therefore, the dressed matrix $\mathbb{J}$ is a regular arrow-bordered circulant one [27]:

$$
\mathbb{J}=\left\{\begin{array}{c|ccccc}
-N \lambda & \varepsilon & \varepsilon & \cdots & \varepsilon & \varepsilon \\
\hline \varepsilon & \lambda & \alpha & \cdots & 0 & \alpha \\
\varepsilon & \alpha & \lambda & \cdots & 0 & 0 \\
\cdots & \ldots & \ldots & \ldots & \ldots & \ldots \\
\varepsilon & \alpha & 0 & \ldots & \alpha & \lambda
\end{array}\right\} .
$$

Its eigenvalues do not depend on $\varepsilon$ and are given as [27]:

$$
\begin{aligned}
j_{ \pm} & =(2 \alpha-(N-1) \lambda \pm \sqrt{\Delta}) / 2, \\
j_{k} & =\lambda+2 \alpha \cos (k \psi), \quad k=1,2, \ldots, N-1,
\end{aligned}
$$

where

$$
\Delta=(2 \alpha+(N+1) \lambda)^{2}+4 N, \quad \text { and } \quad \psi=2 \pi / N
$$

The minimum function $j_{\min }(\lambda)$ is constructed from two of them: $j_{-}(\lambda)$ and $j_{N / 2}(\lambda)$, which have the same value at

$$
j_{\text {cross }}(\alpha)=j_{N / 2}\left(\lambda_{\text {cross }}\right)=-\frac{N\left(8 \alpha^{2}+1\right)}{4(N+1) \alpha} .
$$

Hence, one obtains that $j_{\min }(\lambda)=j_{-}(\lambda)$ for $\lambda \leq \lambda_{\text {cross }}$ and $j_{\min }(\lambda)=j_{N / 2}(\lambda)$ otherwise. The maximum, $\max _{\lambda} j_{\min }(\lambda)$ (and its abscissa) depends on relation between $\lambda_{\text {cross }}$ and $\lambda_{\max }$, where $j_{-}(\lambda)$ reaches its maximum. It can be shown that [27]:

$$
\begin{aligned}
\lambda_{\max }(\alpha) & =-\frac{(N-1)+2 \alpha}{N+1}=-1-\frac{2(\alpha-1)}{N+1}, \\
\max _{\lambda} j_{-}(\lambda) & =\frac{2 N}{N+1}(\alpha-1)=-N\left(\lambda_{\max }+1\right) .
\end{aligned}
$$

Simple algebra shows that $\lambda_{\text {cross }}=\lambda_{\max }$ for $\alpha=1 / 4$, so the final result is

$$
\bar{j}= \begin{cases}\max _{\lambda} j_{-}(\lambda)=\frac{2 N}{N+1}(\alpha-1), & \text { for } \quad \alpha \leq 1 / 4, \\ j_{\text {cross }}(\alpha)=-\frac{2 N}{N+1} \frac{8 \alpha^{2}+1}{8 \alpha}, & \text { for } \quad \alpha \geq 1 / 4\end{cases}
$$

The corresponding abscissa is given as:

$$
\bar{\lambda}= \begin{cases}\lambda_{\max }=-1-\frac{2(\alpha-1)}{N+1}, & \text { for } \quad \alpha \leq 1 / 4, \\ \lambda_{\operatorname{cross}}=\frac{8 \alpha^{2}-N}{4(N+1) \alpha}, & \text { for } \quad \alpha \geq 1 / 4 .\end{cases}
$$

It has to be emphasised that the 'critical' value $\alpha_{\text {crit }}=1 / 4$ is universal - it does not depend on the system size $N$ and a type of couplings with the central spin determined by the sign of $J_{0}$, i.e., by the parameter $\varepsilon$. 


\subsection{The lowest energy configurations}

Since there are $N$ bonds in the outer ring and the same number of couplings between the peripheral spins and the central one (the first and the second term in Eq. (10) or (11), respectively), then it is convenient to divide $E_{\text {LEC }}$ by $N$ and to consider energy density. According to the relation between $E_{\text {LEC }}$ and the eigenvalue $\bar{j}$ (see Sec. 3.2) one obtains:

$$
\frac{E_{\mathrm{LEC}}}{N}= \begin{cases}\alpha-1, & \text { for } \quad \alpha \leq 1 / 4 \\ -\left(\alpha+\frac{1}{8 \alpha}\right), & \text { for } \quad \alpha \geq 1 / 4\end{cases}
$$

The first formula immediately confirms that for $\alpha<1 / 4$ the peripheral spins are ordered ferromagnetically (despite antiferromagnetic couplings for $0<\alpha<1 / 4$ ) and they are antiparallel (parallel) to the central spin $\boldsymbol{s}_{0}$ for $\varepsilon=$ \pm 1 , respectively. Therefore, the collinear LEC, characteristic for non-frustrated systems, retains for relatively weak antiferromagnetic couplings which yield competing interaction, but without non-trivial degeneracy.

In the second range antiferromagnetic ordering of the peripheral spins is preferred: $E_{\mathrm{LEC}} /(N \alpha) \rightarrow-1$ when $\alpha \rightarrow \infty$. For finite $\alpha>1 / 4$ spin vectors with odd (even) indices are rotated by an angle $\varphi$ ( $-\varphi$, respectively) in the same way as it has been given in Sec. 3.1 for $N=2$, i.e., $\cos \varphi=-\varepsilon /(4 \alpha)$. Exchange of spins with odd and even indices (or, equivalently, reversing the sign of $\varphi$ ) yields another LEC for $0<\varphi<\pi$ (see Fig. 3).

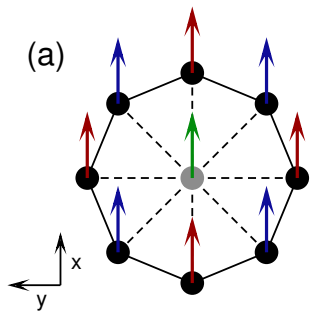

(b)

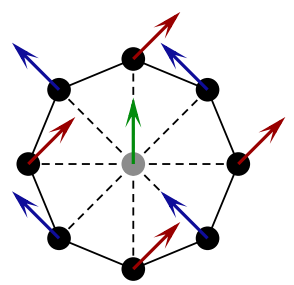

(c)

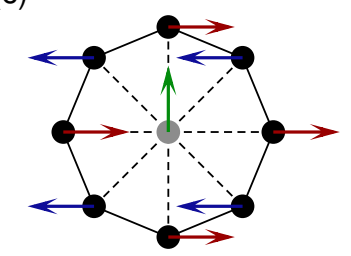

FIG. 3. The LECs for the centred octagon (the wheel $W_{9}$ ) for $\varepsilon=-1$ and (a) $\alpha<1 / 4$, (b) $\alpha=1 / \sqrt{8}$, and (c) $\alpha \rightarrow \infty$. If $\varphi$ is not a multiplicty of $\pi$ (the later two cases) another two LECs are obtained changing sign of the angle $\varphi$. In the case (b) presented LEC corresponds to $\alpha=1 / \sqrt{8}$ and $\varphi= \pm \pi / 4$, when the nearest neighbours are orthogonal.

\section{Conclusions and challenges}

The systems considered in this paper reveal the classical counterpart of the third type frustration defined for quantum systems - in the well-determined range $0<\alpha<1 / 4$ the unique collinear LEC, characteristic for nonfrustrated systems, is observed. Therefore, there exists nontrivial range of the energy function parameter $\alpha$, at which competing interactions are not accompanied by degeneracy. Note that in some approaches frustration is present only in systems with non-trivial degeneracy of the ground state.

The second important phenomenon is universality of the critical value $\alpha_{\text {crit }}=1 / 4$ (cf. [19]) and, moreover, it is also valid in the case of quantum systems $[14,15,35]$. Above this value, the angle $\varphi$ is a continuous function of the parameter $\alpha$.

It should be also emphasized that such general results can be obtained owing to the powerful and efficient approach worked out by Schmidt and Luban. It is a challenging task to apply this method to heterogeneous and/or less symmetric systems. Some initial considerations, including classical analogs of the Ising system with energy function (4), have indicated two facts. At first, in some systems, there is an abrupt change in the LEC, when very weak competing interactions are 'turned on'. Secondly, in the case of rings with antiferromagnetic couplings of the second neighbors the LEC does not change continuously for increasing $\alpha$, but a series of abrupt changes is rather observed.

\section{References}

[1] Furrer A., Waldmann O. Magnetic cluster excitations. Rev. Mod. Phys., 2013, 85 (1), P. 367-420.

[2] Timco G.A., McInnes E.J.L., Winpenny R.E.P. Physical studies of heterometallic rings: An ideal system for studying magnetically-coupled systems. Chem. Soc. Rev., 2013, 42 (4), P. 1796-1806.

[3] McInnes E.J.L., Timco G.A., Whitehead G.F.S., Winpenny R.E.P. Heterometallic rings: Their physics and use as supramolecular building blocks. Angew. Chem. Int. Ed., 2015, 54 (48), 14244.

[4] Ghirri A., Chiesa A., et al. Coherent spin dynamics in molecular $\mathrm{Cr}_{8}$ Zn wheels. The J. Phys. Chem. Lett., 2015,6 (24), 5062.

[5] Baker M.L., Lancaster T., et al. Studies of a large odd-numbered odd-electron metal ring: Inelastic neutron scattering and muon spin relaxation spectroscopy of $\mathrm{Cr}_{8} \mathrm{Mn}$. Chemistry - A European Journal, 2016, 22 (5), P. 1779-1788. 
[6] Woolfson R.J., Timco G.A., et al. $\left[\mathrm{CrF}\left(\mathrm{O}_{2} \mathrm{CtBu}\right)_{2}\right]_{9}$ : Synthesis and characterization of a regular homometallic ring with an odd number of metal centers and electrons. Angew. Chem. Int. Ed., 2016, 55 (31), 8856.

[7] Furukawa Y., Kiuchi K., et al. Evidence of spin singlet ground state in the frustrated antiferromagnetic ring Cr 8 Ni. Phys. Rev. B, 2009, 79 (13), 134416.

[8] Baker M.L., Timco G.A., et al. A classification of spin frustration in molecular magnets from a physical study of large odd-numbered-metal, odd electron rings. Proc. Nat. Acad. Sci. USA, 2012, 109, P. 19113-19118.

[9] Antkowiak M., Kozłowski P., et al. Detection of ground states in frustrated molecular rings by in-field local magnetization profiles. Phys. Rev. $B, 2013,87$ (18), 184430.

[10] Kozłowski P. Frustration and quantum entanglement in odd-membered ring-shaped chromium nanomagnets. Phys. Rev. B, 2015, 91 (17), 174432.

[11] Owerre S.A., Nsofini J. Antiferromagnetic molecular nanomagnets with odd-numbered coupled spins. Europhy. Lett. (EPL), 2015, 110 (4), 47002.

[12] Garlatti E., Bordignon S., et al. Relaxation dynamics in the frustrated $\mathrm{Cr}_{9}$ antiferromagnetic ring probed by NMR. Phys. Rev. B, 2016, 93 (2), 024424.

[13] Kamieniarz G., Florek W., Antkowiak M. Universal sequence of ground states validating the classification of frustration in antiferromagnetic rings with a single bond defect. Phys. Rev. B, 2015, 92 (14), 140411(R).

[14] Florek W., Antkowiak M., Kamieniarz G. Sequences of ground states and classification of frustration in odd-numbered antiferromagnetic rings. Phys. Rev. B, 2016, 94 (22), 224421.

[15] Ako A.M., Waldmann O., et al. Odd-numbered Fe(III) complexes: Synthesis, molecular structure, reactivity, and magnetic properties. Inorg. Chem., 2007, 46 (3), P. 756-766.

[16] Garlatti E., Carretta S., et al. Theoretical design of molecular nanomagnets for magnetic refrigeration. Appl. Phys. Lett., 2013, 103, 202410.

[17] Sharples J.W., Collison D., et al. Quantum signatures of a molecular nanomagnet in direct magnetocaloric measurements. Nature Commun., $2014,5,5321$.

[18] Kakaroni F.E., Collet A., et al. Constructing CrIII-centered heterometallic complexes: [NiII6CrIII] and [CoII6CrIII] wheels. Dalton Trans., 2018, 47 (1), P. 58-61.

[19] Florek W., Kamieniarz G., Marlewski A. Universal lowest energy configurations in a classical Heisenberg model describing frustrated systems with wheel geometry. Phys. Rev. B, 2019, 100 (5), 054434.

[20] Wilson R.J. Introduction to Graph Theory. Addison Wesley Longman Ltd., London, 1996.

[21] Grajek M., Kopyciuk T., Florek W., Marlewski A. Collinear and non-collinear configurations in classical geometrically frustrated ring-shaped systems. Acta Phys. Pol. A, 2018, 133 (3), P. 417-419.

[22] Popov A.P., Anisimov A.V., Eriksson O., Skorodumova N.V. Metastable noncollinear canted states from a phenomenological model of a symmetric ferromagnetic film. Phys. Rev. B, 2010, 81 (5), 054440.

[23] Popov A.P., Rettori A., Pini M.G. Discovery of metastable states in a finite-size classical one-dimensional planar spin chain with competing nearest- and next-nearest-neighbor exchange couplings. Phys. Rev. B, 2014, 90 (13), 134418.

[24] Popov A.P., Rettori A., Pini M.G. Spectrum of noncollinear metastable configurations of a finite-size discrete planar spin chain with a collinear ferromagnetic ground state. Phys. Rev. B, 2015, 92 (2), 024414.

[25] Schmidt H.-J., Luban M. Classical ground states of symmetric Heisenberg spin systems. Journal of Physics A: Math. Gen., 2003, 36 (23), P. 6351-6378.

[26] Schmidt H.-J. Theory of ground states for classical Heisenberg spin systems. Parts I-IV. 2017, arXiv: https://arxiv.org/abs/1701.02489, 1707.02859, 1707.06512, 1710.00318 .

[27] Florek W., Marlewski A. Spectrum of some arrow-bordered circulant matrix. 2019, arXiv: https//arXiv.org/abs/1905.04807.

[28] Toulouse G. Theory of the frustration effect in spin glasses: I. In Mézard M., Parisi G., Virasoro M. A. Spin Glass Theory and Beyond. World Scientific, Singapore: 1987, P. 99-103. Reprinted form: Toulouse G. Communcations de Physique. 1977, 2, P. 115-119.

[29] Vannimenus J., Toulouse G. Theory of the frustration effect: II. Ising spins on a square lattice. J. Phys. C: Solid State Phys., 1977, 10 (18), P. L537-L542.

[30] Pauling L. The structure and entropy of ice and of other crystals with some randomness of atomic arrangement. J. Am. Chem. Soc., 1935, 57 (12), P. 2680-2684.

[31] Wannier G.H. Antiferromagnetism. The triangular Ising net. Phys. Rev., 1950, 79 (2), P. 357-364.

[32] Giampaolo S.M., Gualdi G., Monras A., Illuminati F. Characterizing and quantifying frustration in quantum many-body systems. Phys. Rev. Lett., 2011, 107 (26), 260602.

[33] Binder K., Young A.P. Spin glasses: Experimental facts, theoretical concepts, and open questions. Rev. Mod. Phys., 1986,58 , P. 801-967.

[34] Lacorre P. The constraint functions: an attempt to evaluate the constraint rate inside structures that undergo ordered magnetic frustration. $J$. Phys. C: Solid State Phys., 1987, 20 (29), P. L775-L781.

[35] Florek W., Antkowiak M., Kamieniarz G. The Kahn degenerate frustration points and the Lieb-Mattis level order in heterometallic wheel molecules with competing interactions. J. Mag. Mag. Mater., 2019, 487, 165326. 